\title{
Improving social participation of children with autism spectrum disorder: Pilot testing of an early animal-assisted intervention in Spain
}

\author{
Adriana Ávila-Álvarez $\mathrm{PhD}^{1} \mid$ Miguel Alonso-Bidegain $\mathrm{MD}^{2}$ | Iván De-Rosende- \\ Celeiro $\mathrm{PhD}^{1} \mid$ Mirian Vizcaíno-Cela $\mathrm{MSc}^{1}$ | Lestonnac Larrañeta-Alcalde $\mathrm{MD}^{3} \mid$ \\ Gabriel Torres-Tobío $\mathrm{PhD}^{4}$
}

${ }^{1}$ Occupational Therapy Research Unit in Non-Pharmacological Interventions, University of A Coruña, A Coruña, Spain

${ }^{2}$ Child Rehabilitation and Early Care Unit, A Coruña University Hospital Complex, A Coruña, Spain

${ }^{3}$ Medicine and Speech Therapy, A Coruña, Spain

${ }^{4}$ Department of Physical and Sports Education, University of A Coruña, A Coruña, Spain

\begin{abstract}
Children with autism spectrum disorder (ASD) experience moderate to profound challenges in relation to the skills required for social participation. Animal-assisted intervention (AAI) is increasingly used within early community care. However, the results of its early application in this population group are not known. This pilot study aimed to explore the feasibility of an early intervention based on the use of therapy dogs and to examine their impact on communication and social interaction skills. A within-subject quasi-experimental longitudinal design was used. The instruments for measuring results were the Assessment of Communication and Interaction Skills (ACIS) and Animal-assisted Therapy Flow Sheet, both based on observation. A total of 19 children with confirmed or probable ASD (with mean age of 46.2 months), cared for in a Spanish therapeutic unit, participated in a median of nine AAI sessions, with a mean duration of 19.9 min per session. The total ACIS score increased significantly between the initial and final assessments of the study, with communication and social interaction skills improving with a large effect size. In the Animal-assisted Therapy Flow Sheet instrument, statistically significant improvements were found in most of the items that evaluate the frequency of child-dog social relationships (look at the dog, touch it, talk to it and get involved in an activity with the animal) and child-therapist relationships (look at the therapist and talk to him/her); the effect sizes ranged from medium to large. In conclusion, the early application of an AAI is feasible and seems to improve communication and social interaction skills, both essential elements for social participation. The results suggest that this intervention may be a beneficial non-pharmacological therapy as a complementary approach within community care for children with ASD in the early years of their life.
\end{abstract}

Keywords: Animal assisted therapy; autism spectrum disorder; communication; early intervention; interpersonal relations. 
Autism spectrum disorder (ASD) is a complex neurodevelopmental syndrome. Each person on the spectrum is unique in his or her strengths and abilities. As for the needs profile, one of the key challenges is in social participation, defined as 'involvement in a subset of activities that involve social situations with others' (Bedell, 2012). The performance of these social activities requires the combined use of a set of skills, which have been described by occupational therapists as simple and observable actions with an implicit functional purpose (American Occupational Therapy Association, 2014; Fisher \& Griswold, 2014). Children with ASD experience moderate to profound challenges in relation to the skills required for participation in social life. ASD is usually detected in childhood and diagnosed after the age of three years in most cases (Sheldrick, Maye, \& Carter, 2017).

The early initiation of therapeutic programmes is a basic criterion in the health and social care of children with ASD. Currently, there is a consensus at an international level regarding the benefits of starting intervention as early as possible, due to the existence of greater neuroplasticity in the first years of life (Dawson, 2008; Rogers \& Vismara, 2008). Therapies such as animal-assisted intervention (AAI) are increasingly used as complementary treatment methods within community care. In AAI, interaction with the animal during the performing of activities, in the presence of a therapist, is the central component of the treatment programme. The International Association of Human-Animal Interaction Organizations (IAHAIO) defines AAI as 'a goal-oriented and structured intervention that intentionally includes or incorporates animals in health, education and human service for the purpose of therapeutic gains in humans' (International Association of Human-Interaction Organizations, 2015). Treatment objectives are established for each individual. In the sessions, various types of previously domesticated animals (i.e. trained for social interaction with humans, such as dogs or horses) can participate. This therapy promotes the social interaction of individuals with dementia (Bernabei et al., 2013), reduces stress and pain in hospitalised patients (Bert et al., 2016) and is beneficial for the social skills of adults with mental health problems such as schizophrenia (Lundqvist, Carlsson, Sjödahl, Theodorsson, \& Levin, 2017; Maujean, Pepping, \& Kendall, 2015). Two meta-analyses concluded that AAI improves social functioning and emotional well-being in elderly people and in groups with a chronic health condition, anxiety or depression (Nimer \& Lundahl, 2007; Virués-Ortega, Pastor-Barriuso, Castellote, Población, \& de Pedro-Cuesta, 2012).

The present study addressed the results of an AAI therapy, based on the incorporation of therapy dogs, in a sample of children with ASD. In recent years, several systematic reviews have examined empirical research on the efficacy of AAI in individuals with ASD. In 2013, the review by O'Haire included 14 studies, published between 1989 and 2012, and the majority since 2008; the mean age of participants was 8.1 years and the majority design was that of single-subject research (O'Haire, 2013). Five studies (35.7\%) assessed interventions with therapy dogs, with a mean sample size of 9.4 participants. This review revealed improvements in social interaction and, to a lesser degree, behavioural problems, the severity of the diagnosis and the level of stress. However, it concluded that these results should be interpreted with caution due to the insufficient number of studies included, the small sample size thereof and the existence of several methodological limitations. Subsequently, in 2017, O'Haire assessed the literature on the subject published between 2012 and 2016 (O'Haire, 2017). This review included 28 studies, but only five (17.9\%) analysed treatments with therapy dogs; with respect to this last group, the participants had mean age of 8.8 years, the mean sample size was 3.8 participants and the within-participants studies were the majority design. Although the results were predominantly positive, in relation to social interaction, communication skills, the expression of positive emotions and the degree of stress, the author drew attention to the limitations of the studies, in the samples and at a methodological level, seeing AAI as a 'potentially enriching' therapy and complementary in nature for individuals with ASD. Similarly, in the systematic review by Hallyburton and Hinton (2017), the small sample size of the studies included limited the applicability of the identified findings. It only located two studies on the effects of therapy dogs on the population with ASD; the mean age of the 
participants was 10.2 years and the mean sample size was 12 participants (Hallyburton \& Hinton, 2017).

The literature shows that there is little scientific evidence concerning the incorporation of therapy dogs as a treatment method in children with ASD and the small sample size is one of the key limitations. In addition, although the importance of early intervention has been highlighted, the results of the early implementation of this type of therapy in the study population are not known. Therefore, in this pilot study, we conducted preliminary research on the efficacy of an intervention based on the use of therapy dogs. The first objective was to explore the feasibility of this intervention at the earliest ages. The second was to examine the impact of this AAI on the social participation of children with ASD, through the assessment of its effects on communication and social interaction skills.

\section{METHODS}

\subsection{Study design}

A within-subject quasi-experimental longitudinal design was employed to collect data on the changes that occurred in participants throughout the course of an AAI.

\section{$2.2 \quad$ Setting}

The study reported in this paper was part of a larger research on the effectiveness of an early AAI among children with disabilities. The research was conducted at the Child Rehabilitation and Early Care Unit of the Teresa Herrera Hospital (Coruña), in north-western Spain. This community unit belongs to the public health system and serves a diverse population of families living in urban and rural environments. Its purpose is to provide multidisciplinary therapeutic care and rehabilitation to children with disabilities, who attend the service on an outpatient basis, with special emphasis on prevention and early care during the period between birth and 6 years.

Each child receives individualised and comprehensive attention, specifically in relation to their needs profile for their daily life. The team consists of several health and social professionals, who carry out a periodic follow-up and participate in a coordinated way in the intervention that the child receives. With respect to children with $\mathrm{ASD}$, they receive a treatment programme in the unit, implemented as early as possible and administered 1 or 2 days a week. It comprises a global stimulation therapy in the different areas of development, for 45 min each day.

\subsection{Participants}

This study was conducted between April and November 2016; the research was interrupted during August as this was a holiday period. Participants were outpatients seen in the unit. All children with confirmed or probable ASD who received therapeutic care in the unit during the study period were invited to participate. The inclusion criteria were as follows: (a) children living in the community aged between 30 months and 6 years; (b) with a probable or confirmed primary diagnosis of ASD, provided by a specialist physician based on the criteria of the Diagnostic and Statistical Manual of Mental Disorders, Fifth Edition (American Psychiatric Association, 2013); (c) possessing the mental and physical capacity to understand and follow intervention procedures, in accordance with the assessment by the unit's specialist physician; (d) no known history of cynophobia or allergy to dogs; (e) not having previously participated in therapy sessions assisted by dogs; and (f) not receiving non-pharmacological therapeutic care at a treatment centre other than the study unit. We excluded children with asthma, respiratory disease with an obstructive 
component, in immunosuppression and all those who showed excessive discomfort, anxiety or fear when performing activities with the dog during intervention. We also excluded all those children who did not receive at least five AAI sessions throughout the study period, given that this is considered the minimum intervention dose required to observe clinically relevant results, in accordance with our previous experience in relationship to this therapeutic modality.

The study protocol was approved by the regional ethics committee (Research Ethics Committee of Coruña-Ferrol). The parents or legal guardians of each child received verbal and written information about the research. They were informed that participation was voluntary and that they could withdraw their child at any time. Subsequently, they signed an informed consent form to authorise their child's participation in the study. The confidentiality of the participants was preserved in accordance with the Spanish Data Protection Law. Table 1 shows the information collected at the beginning of the study in order to describe the characteristics of the sample.

Table 1. Characteristics of the participating children at initial assessment $(n=19)$

\begin{tabular}{ll}
\hline Sample characteristics & Value, $\mathrm{n}(\%)$ \\
\hline Autism spectrum disorder & \\
Confirmed diagnosis & $15(79)$ \\
Probable diagnosis & $4(21)$ \\
Age (months) & \\
Mean (SD) & $46.2(12.6)$ \\
Range & $30-66$ \\
Gender & $13(68.4)$ \\
Male & \\
Secondary diagnosis & $4(21.1)$ \\
Attention deficit hyperactivity disorder & \\
Verbal ability & $4(21.1)$ \\
Presence of verbal language & \\
Pet ownership & $8(42.1)$ \\
Yes & $7(36.8)$ \\
Dog & $3(15.8)$ \\
Cat & \\
\hline
\end{tabular}

${ }^{a}$ As defined by the Autism Diagnostic Interview-Revised (Lord et al., 1994).

\section{$2.4 \quad$ Intervention}

The intervention is a complementary therapeutic modality, designed to encourage the social participation and well-being of children with ASD, through the acquisition and improvement of psychosocial skills and the promotion of behaviours and interactions of a social nature. It uses the relationship and activity with the dog as an intervention method, with the presence and mediation of a specialised therapist. It deliberately introduces a trained dog into sessions with specific objectives defined for each participant. 
The sessions were individual in nature, to promote animal welfare. They were held one day a week, with an approximate duration of $20 \mathrm{~min}$. One of the factors that had a bearing on interaction time was the creation and consolidation of the child-dog bond, whose development was progressive in most of the participants. Therefore, the tendency towards a longer duration was observed as the study progressed. The number of sessions was variable among the participants, due to factors such as not attending therapy due to illness, the family's change of residence or being discharged from the unit. In all sessions, a therapy dog, a therapist and the participant's parent or guardian were present. The sessions were conducted by an occupational therapist with specialised training in AAI. In the child-dog interaction, the therapist intervened as mediator and facilitator, also encouraging the active participation of parents in the activities. The child's interest in the dog was encouraged. Communication and social interaction were promoted among the child, the dog, therapist and tutor, providing feedback, initiating the social bond, participating in the activities or responding to the social behaviour of the child or tutor. Moreover behavioural strategies of modelling and reinforcement were used. Five therapy dogs (four males and one female) were used, with mean age of 4 years: three Labrador Retrievers (large size), one Galician Shepherd Dog (large size) and one Spanish Water Dog breed (medium size). The animal was trained in order to adapt easily to different situations, be friendly with children and have an obedient and calm temperament. All dogs were trained as therapy dogs and introduced into the unit by registered dog trainers from a specialised external centre (Montegatto). IAHAIO standards were followed (International Association of Human-Interaction Organizations, 2015). The animals were examined periodically by an authorised veterinarian, ensuring that they were in good health and met basic hygiene standards.

The intervention was provided in a $28 \mathrm{~m}^{2}$ room, with natural lighting from several windows. A semi-standardised approach was followed, characterised by designing the therapeutic activities based on the needs and interests of each child. The activities were simple and interactive, and the involvement of the child in the selection thereof was promoted to encourage their motivation and interest. In the initial sessions, the therapist presented the repertoire of intervention activities to the child (adult-directed sessions). Subsequently, through verbal, gestural and pictographic prompts, the therapist promoted a gradual increase in the child's participation in the choice of session activities, in order that the child can choose how to structure the session time based on individual preferences. Therefore, the child was encouraged to have the greatest possible control over the choice of activities. To communicate what he/she wanted to do, the participants used vocalisations, gestures, exchange of pictures or another form of communication. In addition, each child was allowed to initiate free play moments with the therapy dog (child-initiated activities).

Various types of activities were used; in brief, they focused on the knowledge of the dog, interaction with the dog, the care of the animal and playful occupations with a primary focus of a social nature (see Table S1). In the earliest sessions, the therapist encouraged exploration and bonding with the dog, introducing the animal and encouraging the child to have visual, verbal and tactile contact with the dog by speaking to it and through gestures, such as pointing out the parts of its body, asking for the dog to give its paw, and stroking, hugging or snuggling with the animal. Later on, the child was introduced to different types of tasks related to the responsibilities involved with dog care, such as brushing, feeding and providing the dog with water or taking it for walks. Finally, various games were implemented that required the child's interaction with the animal and with the people present in the session, to encourage contextually appropriate social skills, such as cooperating in a common task, sharing materials, respecting turns, making requests and fulfilling these, expressing enjoyment through smiling or embracing. As the intervention progressed, the animal care activities were combined with those that were playful in approach. 
Two standardised instruments were used as outcome measures. The ACIS, version 4.0 (Forsyth, Lai, \& Kielhofner, 1999; Forsyth, Salamy, Simon, \& Kielhofner, 1998), was the primary result. This observation-based tool systematically assesses communication and social interaction skills, which are defined as observable actions used to express intentions or needs and to interact successfully with other people in occupations and social contexts (Forsyth et al., 1999). It includes 20 items distributed in three domains: physicality, information exchange and relations. Each item is scored on a 4-point Likert scale (1-4): 1 represents a severe deficit that causes an unacceptable delay or breakdown of interaction, and 4 a competent skill that supports the continuity of social interaction. The total score is obtained by adding the scores of all the items, ranging from 20 (severe deficit) and 80 points (competent performance). This scale has acceptable internal and construct validity and good reliability (Forsyth et al., 1999). It reliably separates individuals with different types of disabilities into six skill levels. The total score allows different diagnostic groups to be individuated, ordering them from higher to lower degree of skills acquisition, following a logical pattern: in a sample of individuals with disabilities with different health conditions, participants with depression obtained the highest scores, while individuals with ASD had the most severe deficits (Forsyth et al., 1999). In our research, a trained occupational therapist applied this scale in the study unit, in the initial (baseline stage) and final assessments. As the recommended observation time for using the scale ranges from 15 to $45 \mathrm{~min}$, each assessment was performed for two days. The initial evaluation consisted of observing the child in the first two AAI sessions. The same procedure was followed for the final assessment, carried out in the final two sessions.

As a secondary data collection instrument, a tool specifically validated to evaluate social behaviour during AAI sessions was used: Animal-assisted Therapy Flow Sheet (Richeson \& McCullough, 2002, 2003). This scale enables the effects of the AAI in the area of social participation to be analysed and detects the changes that occur throughout the sessions in the child's relationships with the therapy dogs and with the therapists. It is a brief questionnaire, consisting of nine items, with questions about the frequency of child-dog social relationships (six items) and client-therapist relationships (three items). It uses Likert-type responses, with four points: 1 point (never), 2 (once), 3 (two or three times) and 4 (several times). It displays appropriate levels of content validity and reliability (Richeson \& McCullough, 2002). In a sample of individuals living in long-term care facilities, the inter-observer reliability was high (0.98) and a Cronbach's alpha of 0.78 was obtained, demonstrating good internal consistency (Richeson \& McCullough, 2002). It is applied over an approximate time period of $5 \mathrm{~min}$, is easy to use and its use requires no specialised training. In our study, this scale was applied through direct observation of AAI sessions by a health professional with experienced in the assessment of children with ASD. For data analysis, the scores obtained in the first session $\left(T_{1}\right)$, the session taking place in the middle of the intervention $\left(T_{2}\right)$ and the last session $\left(T_{3}\right)$ were used.

\subsection{Data analysis}

Descriptive statistics were used to summarise the results. The categorical variables were described through frequencies and percentages. The variables that followed a normal distribution were described using the mean and the standard deviation $(S D)$; those that did not follow the normal distribution and the ordinal variables, through the median and the first and third quartiles ( $Q 1-Q 3)$. Participants were divided into two groups (pet ownership vs. no). The total scores of the ACIS (in the initial and final assessments) and the Animal-assisted Therapy Flow Sheet (in the $T_{1}, T_{2}$ and $T_{3}$ assessments) were compared between these two groups, using the MannWhitney $U$-test. 
The changes in the scores on the ACIS between the initial (baseline) and final assessments were tested for significance by means of the Wilcoxon's signed-rank non-parametric test. Friendman's non-parametric analyses were conducted to test for differences in the items of the Animal-assisted Therapy Flow Sheet from $T_{1}$ to $T_{3}$, and the Wilcoxon's test was used as posthoc procedure.

Bonferroni corrections for multiple comparisons (0.05/3) were applied to prevent type I errors. The effect size $(r)[\mathrm{ES}(r)]$ of the changes in scores on the ACIS and the Animal-assisted Therapy Flow Sheet items was calculated by dividing the $Z$ of the Wilcoxon's tests by the square root of the total number of observations. An ES $(r)$ of 0.10 constitutes a small effect, 0.30 medium effect and 0.50 large effect (Cohen, 1988). For all tests except the Wilcoxon posthoc test, the level of significance was set a priori at $p<.05$ (two-sided). The IBM SPSS 22.0 was used for the statistical analysis.

During the study period, a total of 23 children met the inclusion criteria and all parents or guardians authorised their participation in the research. Subsequently, no participant was withdrawn from the study at the request of the parents/guardian, nor owing to excessive discomfort, anxiety or fear during the intervention sessions. Of the 23 children initially enrolled, four participants (17.4\%) were excluded due to taking part in less than five sessions; in all cases, the reason that prevented them completing the minimum number of sessions established was their being discharged from the unit. Therefore, the final sample consisted of 19 participants. At the start of the study, $79 \%$ of the sample had an ASD diagnosis. At the beginning of the study, $21 \%$ of the participants had a probable diagnosis of ASD; with respect to this last group, all children received a definitive diagnosis of ASD before completing this investigation. Table 1 describes the main characteristics of the sample. The mean age was 46.2 months (SD 12.6). One fifth of the participants displayed verbal language, as per the definition of verbal language in the Autism Diagnostic Interview-Revised (Lord, Rutter, \& Le Couteur, 1994). Eight participants had at least one dog or cat in their home (pet ownership group), and the remaining participants did not have any pets. Regarding the total scores of the ACIS and the Animal-assisted Therapy Flow Sheet, no significant differences were found between these two groups.

\subsection{AAI sessions}

In relation to the intervention analysed, the participating children received a median of nine sessions $(Q 1-Q 3$ 8-12) during the study period. The mean duration of the sessions was 19.9 min (SD 5.8). The participants did not attend a mean of 2.2 sessions (SD 1.9).

\subsection{Results in the ACIS tool}

Statistically significant changes were found in the ACIS score between the initial and final assessments: the median of the total score increased seven points, going from 24 to 31 points $(p<.001)$, so that communication and interaction skills improved, with a large effect size [ES $(r)=0.62]$ (Table 2). With respect to the 20 items of the ACIS, Table 2 shows the changes in the scores between the baseline of the study and the final assessment. The score improved significantly in a total of 12 items and the effect size ranged from medium [ES $(r)=0.43$ ] to large [ES $(r)=0.88]$. 
Table 2. Communication and interaction skills at the baseline stage and the final assessment $(n=19)$

\begin{tabular}{|c|c|c|c|c|}
\hline & Baseline stage & Final assessment & $p$-Value & Effect size \\
\hline & Median $(Q 1-Q 3)$ & Median $(Q 1-Q 3)$ & & \\
\hline \multicolumn{5}{|l|}{ Physicality } \\
\hline Contacts & $1(1-2)$ & $2(2-3)$ & $p<.001 *$ & 0.86 \\
\hline Gazes & $2(1-2)$ & $2(2-2)$ & $.003 *$ & 0.68 \\
\hline Gestures & $1(1-2)$ & $2(1-2)$ & $.008^{*}$ & 0.43 \\
\hline Manoeuvres & $1(1-2)$ & $2(2-3)$ & $p<.001 *$ & 0.86 \\
\hline Orients & $2(1-2)$ & $2(2-3)$ & $p<.001 *$ & 0.88 \\
\hline Postures & $1(1-2)$ & $1(1-2)$ & .083 & - \\
\hline \multicolumn{5}{|c|}{ Information exchange } \\
\hline Articulates & $1(1-2)$ & $2(1-2)$ & $.046^{*}$ & 0.45 \\
\hline Asserts & $1(1-1)$ & $1(1-2)$ & .157 & - \\
\hline Asks & $1(1-1)$ & $1(1-1)$ & .157 & - \\
\hline Engages & $1(1-2)$ & $2(1-2)$ & $.046^{*}$ & 0.45 \\
\hline Expresses & $1(1-2)$ & $2(1-2)$ & $.001 *$ & 0.75 \\
\hline Modulates & $1(1-2)$ & $2(1-2)$ & .083 & - \\
\hline Shares & $1(1-2)$ & $1(1-2)$ & .084 & - \\
\hline Speaks & $1(1-1)$ & $2(1-2)$ & $.003 *$ & 0.68 \\
\hline Sustains & $1(1-1)$ & $1(1-1)$ & .317 & - \\
\hline \multicolumn{5}{|l|}{ Relations } \\
\hline Collaborates & $1(1-2)$ & $1(1-2)$ & $.034 *$ & 0.48 \\
\hline Conforms & $1(1-2)$ & $2(2-2)$ & $.001 *$ & 0.75 \\
\hline Focuses & $1(1-2)$ & $2(2-2)$ & $.001 *$ & 0.79 \\
\hline Relates & $1(1-2)$ & $1(1-2)$ & .317 & - \\
\hline Respects & $1(1-1)$ & $1(1-1)$ & .317 & - \\
\hline Total score & $24(21-30)$ & $31(28-40)$ & $p<.001 *$ & 0.62 \\
\hline
\end{tabular}

Note: Values based on the Assessment of Communication and Interaction Skills (ACIS). An increase in score indicates an improved skill.

$Q 1-Q 3$, first and third quartile.

* Indicates significant finding $(p<.05)$.

\subsection{Results in the Animal-assisted Therapy Flow Sheet instrument}

Table 3 presents changes in the items of this instrument during the study period. One item (remembered dog handler's name) did not reach statistical significance. Friedman's analyses showed a statistically significant change in eight items from $T_{1}$ to $T_{3}$ times. 
Table 3. Social behaviour of the participating children during the animal-assisted intervention $(n=19)$

\begin{tabular}{|c|c|c|c|c|c|c|c|}
\hline & \multicolumn{2}{|l|}{$T_{1}$} & \multicolumn{2}{|l|}{$T_{2}$} & \multicolumn{2}{|l|}{$T_{3}$} & \multirow[b]{2}{*}{$\begin{array}{l}p \text { - } \\
\text { Value }\end{array}$} \\
\hline & $\begin{array}{l}\text { Mean } \\
\text { rank }^{\mathrm{a}}\end{array}$ & $\begin{array}{l}\text { Median } \\
(Q 1-Q 3)\end{array}$ & $\begin{array}{l}\text { Mean } \\
\text { rank }^{\mathrm{a}}\end{array}$ & $\begin{array}{l}\text { Median } \\
(Q 1-Q 3)\end{array}$ & $\begin{array}{l}\text { Mean } \\
\text { rank }^{\mathrm{a}}\end{array}$ & $\begin{array}{l}\text { Median } \\
(Q 1-Q 3)\end{array}$ & \\
\hline Looked at dog & 1.63 & $4(3-4)$ & 2.21 & $4(4-4)$ & 2.16 & $4(4-4)$ & $.005^{*}$ \\
\hline Touched dog & 1.37 & $2(1-3)$ & 2.21 & $3(2-4)$ & 2.42 & $4(2-4)$ & $<.001 *$ \\
\hline Spoke to dog & 1.71 & $1(1-1)$ & 2.03 & $1(1-1)$ & 2.26 & $1(1-2)$ & $.005^{*}$ \\
\hline $\begin{array}{l}\text { Remembered and } \\
\text { used dog's name }\end{array}$ & 1.76 & $1(1-1)$ & 2.05 & $1(1-1)$ & 2.18 & $1(1-2)$ & $.048^{*}$ \\
\hline $\begin{array}{l}\text { Engaged in } \\
\text { activity with dog }\end{array}$ & 1.37 & $1(1-1)$ & 2.13 & $2(1-3)$ & 2.50 & $2(1-3)$ & $<.001 *$ \\
\hline $\begin{array}{l}\text { Reminisced about } \\
\text { own dog }\end{array}$ & 1.74 & $1(1-1)$ & 2.05 & $1(1-1)$ & 2.21 & $1(1-2)$ & $.009 *$ \\
\hline $\begin{array}{l}\text { Looked at dog } \\
\text { handler }\end{array}$ & 1.74 & $4(3-4)$ & 1.82 & $4(3-4)$ & 2.45 & $4(4-4)$ & $.001 *$ \\
\hline $\begin{array}{l}\text { Spoke to dog } \\
\text { handler }\end{array}$ & 1.66 & $1(1-1)$ & 1.97 & $1(1-2)$ & 2.37 & $1(1-3)$ & $.001 *$ \\
\hline $\begin{array}{l}\text { Remembered dog } \\
\text { handler's name }\end{array}$ & 1.84 & $1(1-1)$ & 2.08 & $1(1-1)$ & 2.08 & $1(1-1)$ & .105 \\
\hline
\end{tabular}

Note: Values based on the Animal-assisted Therapy Flow Sheet. A higher score means a higher frequency of the social behaviour.

$T_{1}$, first session; $T_{2}$, session in the middle of the intervention; $T_{3}$, final session; $Q 1-Q 3$, first and third quartile.

${ }^{a}$ Friedman's test.

* Indicates significant finding $(p<.05)$.

Regarding these eight items, Table 4 presents the level of significance and the ES( $r)$ of the changes identified in the posthoc procedures. Statistically significant improvements were found in six items, with effect sizes that ranged from medium $[\mathrm{ES}(r)=0.39]$ to large $[\mathrm{ES}(r)=0.52]$. The changes in two items did not reach statistical significance (remembered and used dog's name, and reminisced about own dog). 
Table 4. Changes in the social behaviour of participants during the intervention: posthoc procedures $(n=19)$

\begin{tabular}{|c|c|c|c|c|c|c|}
\hline \multirow[t]{2}{*}{ Animal-assisted Therapy Flow Sheet ${ }^{a}$} & \multicolumn{2}{|c|}{$T_{1}$ versus $T_{2}$} & \multicolumn{2}{|c|}{$T_{2}$ versus $T_{3}$} & \multicolumn{2}{|c|}{$T_{1}$ versus $T_{3}$} \\
\hline & $p$-Value & $\mathrm{ES}(r)$ & $\mathrm{ES}(r)$ & $\mathrm{ES}(r)$ & $p$-Value & $\mathrm{ES}(r)$ \\
\hline Looked at dog & $.011^{*}$ & 0.41 & .564 & - & $.003 *$ & 0.49 \\
\hline Touched dog & $.016^{*}$ & 0.39 & .141 & - & $.005^{*}$ & 0.46 \\
\hline Spoke to dog & .059 & - & .102 & - & $.015^{*}$ & 0.39 \\
\hline Remembered and used dog's name & .063 & - & .157 & - & .023 & - \\
\hline Engaged in activity with dog & $.002 *$ & 0.50 & .038 & - & $.001 *$ & 0.52 \\
\hline Reminisced about own dog & .063 & - & .157 & - & .023 & - \\
\hline Looked at dog handler & .655 & - & $.005 *$ & 0.46 & $.003 *$ & 0.49 \\
\hline Spoke to dog handler & .059 & - & .038 & - & $.006^{*}$ & 0.45 \\
\hline
\end{tabular}

Note: Values based on the Animal-assisted Therapy Flow Sheet. A higher score means a higher frequency of the social behaviour.

$T_{1}$, first session; $T_{2}$, session in the middle of the intervention; $T_{3}$, final session; $Q 1-Q 3$, first and third quartile. ${ }^{a}$ Friedman's test.

* Indicates significant finding $(p<.05)$.

Regarding these eight items, Table 4 presents the level of significance and the ES $(r)$ of the changes identified in the posthoc procedures. Statistically significant improvements were found in six items, with effect sizes that ranged from medium $[\mathrm{ES}(r)=0.39]$ to large $[\mathrm{ES}(r)=0.52]$. The changes in two items did not reach statistical significance (remembered and used dog's name, and reminisced about own dog). 
Table 4. Changes in the social behaviour of participants during the intervention: posthoc procedures $(n=19)$

\begin{tabular}{|c|c|c|c|c|c|c|}
\hline \multirow[t]{2}{*}{ Animal-assisted Therapy Flow Sheet ${ }^{a}$} & \multicolumn{2}{|c|}{$T_{1}$ versus $T_{2}$} & \multicolumn{2}{|c|}{$T_{2}$ versus $T_{3}$} & \multicolumn{2}{|c|}{$T_{1}$ versus $T_{3}$} \\
\hline & $p$-Value & $\mathrm{ES}(r)$ & $p$-Value & $\mathrm{ES}(r)$ & $p$-Value & $\mathrm{ES}(r)$ \\
\hline Looked at dog & $.011 *$ & 0.41 & .564 & - & $.003^{*}$ & 0.49 \\
\hline Touched dog & $.016^{*}$ & 0.39 & .141 & - & $.005^{*}$ & 0.46 \\
\hline Spoke to dog & .059 & - & .102 & - & $.015^{*}$ & 0.39 \\
\hline Remembered and used dog's name & .063 & - & .157 & - & .023 & - \\
\hline Engaged in activity with dog & $.002 *$ & 0.50 & .038 & - & $.001 *$ & 0.52 \\
\hline Reminisced about own dog & .063 & - & .157 & - & .023 & - \\
\hline Looked at dog handler & .655 & - & $.005 *$ & 0.46 & $.003^{*}$ & 0.49 \\
\hline Spoke to dog handler & .059 & - & .038 & - & $.006^{*}$ & 0.45 \\
\hline
\end{tabular}

Note: $T_{1}$, first session; $T_{2}$, session in the middle of the intervention; $T_{3}$, final session; ES $(r)$, effect size.

${ }^{a}$ The items with statistically significant changes from $T_{1}$ to $T_{3}$ sessions.

* Indicates significant finding after Bonferroni correction $(p<.017)$.

4

DISCUSSION

The main contribution of our study was to examine the results of early implementation of an AAI in children with ASD. This is the first research specifically designed to explore the impact of the use of therapy dogs when these children are younger in age. Other contributions were the inclusion of a greater number of participants than in the previous literature, thus addressing one of the principal limitations of the evidence in this field (O'Haire, 2013, 2017), and the enrichment of the results by measuring effect sizes, this representing a strength uncommon in previous studies (O'Haire, 2017). Factors such as the consent of all the invited parents, the recruitment of a sample of close to 20 participants in a small geographic area and in a relatively short period, as well as the absence of withdrawals from the research owing to expressions of fear or excessive anxiety in the presence of the dog, suggested the acceptability of this therapeutic modality. These findings demonstrate that its application is feasible in the study population. In addition, they represent a promising line of work, given that the participants improved considerably in most of the communication and social interaction skills analysed.

The participating children showed very important limitations in the skills observed by the ACIS. However, the results revealed that the IAA had a positive effect on the total score of this scale, finding a large improvement after the therapeutic programme. The greatest gains were located in the skills related to the use of the body in social interaction: directing the head towards other individuals, moving the body to relate to others and establishing/accepting physical contact. In social relationship skills, maintaining attention focused on interaction and fulfilling social norms were the main improvements; in the domain of exchange of information, the main advance was registered in the expression of affection. The Animal-assisted Therapy Flow Sheet corroborated the improvement of the sample in terms of eye and verbal contact between child and therapist. Using this tool also enabled us to analyse what the changes in the child-dog relationship were, another aspect of interest, as the verbal and non-verbal behaviours of the child towards the dog have been included within the definition of social interaction from the outset of research on AAI (Redefer \& Goodman, 1989). Our study identified a significant increase in the frequency of the child's eye, verbal and physical contact with the animal, as well as his/her participation in activities 
with the dog. The positive impact of AAI on communication and social interaction skills is consistent with the limited evidence currently available on interventions with therapy dogs in children with ASD (Funahashi, Gruebler, Aoki, Kadone, \& Suzuki, 2014; Fung, 2015; Grigore \& Rusu, 2014; Martin \& Farnum, 2002; Redefer \& Goodman, 1989; Sams, Fortney, \& Willenbring, 2006; Silva, Correia, Lima, Magalhães, \& de Sousa, 2011). However, it is difficult to compare these findings with the previous literature due to the existence of considerable differences in study designs and methodologies, the diversity of samples and the heterogeneity of assessment instruments. Furthermore, in order to design a therapeutic programme with optimal and, at the same time, efficient results, with the lowest possible cost, our intervention was relatively short compared to other research in this field, including fewer sessions (Fung, 2015; Fung \& Leung, 2014; Martin \& Farnum, 2002; Redefer \& Goodman, 1989) and with a shorter duration (Funahashi et al., 2014; Grigore \& Rusu, 2014; Sams et al., 2006; Silva et al., 2011), which entails a shorter period of exposure to the dog.

Our study is in line with current evidence in the field of human-animal interaction, showing the benefits that dogs provide in therapeutic contexts. Possible explanations have been suggested to argue these positive effects. Humans seem to show a natural interest in animals. It has been consistently observed that children with ASD speak significantly more about dogs than about objects or toys, and are happier in the presence of the animal (Martin \& Farnum, 2002). When they can choose freely with whom to interact, they relate more to animate beings than to toys, opting more for dogs than for contact with people (Prothmann, Ettrich, \& Prothmann, 2009). In other research, it was found that these children smile more frequently in sessions with dogs than in those in which only the therapist is present (Stevenson, Jarred, Hinchcliffe, \& Roberts, 2015). Dogs have also developed a natural tendency towards relationships with human beings, proving themselves to be friendly and offering continuous unconditional support (Fine, 2010; Friesen, 2010). In addition, the dog's behaviour consists of non-verbal actions, based on the use of the body, simple, predictable and interpretable for the sample more easily than the diverse and complex range of human reactions and intentions (Berry, Borgi, Francia, Alleva, \& Cirulli, 2013; Prothmann, Ettrich, \& Prothmann, 2009; Redefer \& Goodman, 1989). Therefore, for children with ASD, the relationship with the dog is less challenging; this encourages the development of a calm therapeutic environment (Friesen, 2010). Similarly, a study of individuals with ASD showed that interaction with dogs is useful for the management of stress, with relevant results at the neuroendocrine level, since it significantly reduces cortisol levels (Viau et al., 2010). The construction of a relaxing, familiar area could motivate the child to communicate and initiate activities with people, contributing to a greater understanding of his/her surrounding environment, as well as to the acquisition and training of social skills.

These results should be interpreted with caution due to the following limitations. The principal limitation was the absence of a control group, so it is not possible to ascertain the influence on the results of other treatments that participants received, such as pharmacological therapy. A second limitation was the lack of information on potentially important data for the study's findings, such as the severity of the diagnosis. The participants were a non-probability convenience sample. The sample consisted of children with ASD receiving treatment in a single therapeutic unit, of reference for a small geographical area, recruited by a non-random technique and predominantly male participants, owing to which our results may not be generalisable for the different types of treatment settings for ASD in Spain or for all children with this health condition in the ages analysed.

Another limitation was the inclusion of children with probable ASD. Most children are referred to the unit with the diagnosis of ASD previously confirmed by specialist physicians or multidisciplinary teams. When children are referred with probable ASD, the unit's specialist physician conducts a comprehensive diagnostic assessment, implemented during a period of time ranging from one to several weeks, through multiple sources of information (e.g. interviews, standardised instruments, assessment of co-occurring health conditions, observation in different settings and report from the pre-school or school). However, to comply with the key principle of 
early access to therapeutic care, children with probable ASD begin therapy quickly after referral, including the intervention analysed in this investigation, so they usually begin the AAI before completing the diagnostic assessment. It should be noted that all participants received a definitive diagnosis of ASD before the end of the study. Another limitation to consider is the variability in the number and length of AAI sessions. Finally, this study did not include a post-intervention follow-up. Although the benefits were substantial, conducting follow-up assessments over a long period of time enables the extent to which these positive findings are maintained after the end of the study to be determined.

More research is required in order to overcome the limitations mentioned above and validate the results of our pilot study. The next step should be to conduct a randomised controlled trial, with long-term monitoring, that explores the impact of dog-assisted therapy in children with ASD at the earliest ages. As a comparison group, it could include children from the same study population, but who are on the waiting list to receive AAI. In addition, although our research substantially exceeded the average sample size of the previous literature (Hallyburton \& Hinton, 2017; O'Haire, 2013, 2017), large-scale experimental designs should be used, with a larger sample from different therapeutic units in the country, to favour the generalisability of the results and provide a stronger evidence basis for the effectiveness of this intervention.

In conclusion, this research reveals that the early application of an intervention based on therapy dogs is feasible in children with ASD and seems to significantly improve communication and social interaction skills, both seriously compromised in the study population. As these are essential factors for daily performance in social life, this intervention could act as a facilitator of social participation. The results indicate the positive impact of AAI in the area of social functioning, suggesting that it may be a beneficial non-pharmacological therapy as a complementary approach within community care for children with ASD in the first years of their life.

\section{ACKNOWLEDGEMENTS}

The authors gratefully acknowledge financial support provided by the Fundación María José Jove (A Coruña, Spain). We thank the following organisations for their collaboration in this research: Fundación María José Jove, Fundación Profesor Novoa Santos, Fundación Universidade da Coruña and Centro Canino Montegatto. The authors thank the therapists, children, parents and legal guardians who took the time to participate in this study.

\section{CONFLICT OF INTEREST}

No conflicts of interest have been declared. 


\section{REFERENCES}

American Psychiatric Association. (2013). Diagnostic and statistical manual of mental disorders (5th ed.). Whashington, DC: American Psychiatric Association.

American Occupational Therapy Association. (2014). Occupational therapy practice framework: Domain and process. American Journal of Occupational Therapy, 68(Suppl. 1), S1S48. https://doi.org/10.5014/ajot.2014.682006

Bedell, G. M. (2012). Measurement of social participation. In V. Anderson, \& M. H. Beauchamp (Eds.), Developmental social neuroscience and childhood brain insult: Theory and practice (pp. 184- 206). New York, NY: Guilford Press.

Bernabei, V., De Ronchi, D., La Ferla, T., Moretti, F., Tonelli, L., Ferrari, B., ... Atti, A. R. (2013). Animal-assisted interventions for elderly patients affected by dementia or psychiatric disorders: A review. Journal of Psychiatric Research, 47, 762773. https://doi.org/10.1016/j.jpsychires.2012.12.014

Berry, A., Borgi, M., Francia, N., Alleva, E., \& Cirulli, F. (2013). Use of assistance and therapy dogs for children with autism spectrum disorders: A critical review of the current evidence. Journal of Alternative and Complementary Medicine, 19(2), 73- 80. https://doi.org/10.1089/acm.2011.0835

Bert, F., Gualano, M. R., Camussi, E., Pieve, G., Voglino, G., \& Siliquini, R. (2016). Animal assisted intervention: A systematic review of benefits and risks. European Journal of Integrative Medicine, 8(5), 695- 706. https://doi.org/10.1016/j.eujim.2016.05.005

Cohen, J. (1988). Statistical power analysis for the behavioral sciences ( 2 nd ed.). Hillsdale, NJ: Erlbaum.

Dawson, G. (2008). Early behavioral intervention, brain plasticity, and the prevention of autism spectrum disorder. Development and Psychopathology, 20(3), 775803. https://doi.org/10.1017/S0954579408000370

Fine, A. H. (2010). Handbook on animal-assisted therapy theoretical foundations and guidelines for practice ( 3 rd ed.). Burlington, MA: Elsevier.

Fisher, A. G., \& Griswold, L. A. (2014). Performance skills: Implementing performance analyses to evaluate quality of occupational performance. In B. A. Boyt, G. Gillen, \& M. Scaffa (Eds.), Willard and Spackman's occupational therapy (12th ed.) (pp. 249264). Philadelphia, PA: Lippincott Willians \& Wilkins.

Forsyth, K., Lai, J., \& Kielhofner, G. (1999). The assessment of communication and interaction Skills (ACIS): Measurement properties. British Journal of Occupational Therapy, 62(2), 6974. https://doi.org/10.1177/030802269906200208

Forsyth, K., Salamy, M., Simon, S., \& Kielhofner, G. (1998). The assessment of communication and interaction skills (Version 4.0). Chicago, IL: University of Illinois at Chicago.

Friesen, L. (2010). Exploring animal assisted programs with children in school and therapeutic contexts. Early Childhood Education Journal, 37(4), 261- 267. https://doi.org/10.1007/s10643009-0349-5

Funahashi, A., Gruebler, A., Aoki, T., Kadone, H., \& Suzuki, K. (2014). Brief report: The smiles of a child with autism spectrum disorder during an animal-assisted activity may facilitate social positive behaviors - Quantitative analysis with smile-detecting interface. Journal of Autism and Developmental Disorders, 44(3), 685- 693. https://doi.org/10.1007/s10803-013-1898-4

Fung, S. C. (2015). Increasing the social communication of a boy with autism using animal-assisted play therapy: A case report. Advances in mind-body Medicine, 29(3), 27-31.

Fung, S. C., \& Leung, A. S. M. (2014). Pilot study investigating the role of therapy dogs in facilitating social interaction among children with autism. Journal of Contemporary Psychotherapy, 44(4), 253-262. https://doi.org/10.1007/s10879-014-9274-z

Grigore, A. A., \& Rusu, A. S. (2014). Interaction with a therapy dog enhances the effects of social story method in autistic children. Society and Animals, 22(3), 241261. https://doi.org/10.1163/15685306-12341326

Hallyburton, A., \& Hinton, J. (2017). Canine-assisted therapies in autism: A systematic review of published studies relevant to recreational therapy. Therapeutic Recreation Journal, 51(2), $127-$ 142. https://doi.org/10.18666/TRJ-2017-V51-I2-7969

International Association of Human-Interaction Organizations. (2015). The IAHAIO definitions for animal-assisted intervention and guidelines for wellness of animals involved, IAHAIO White Paper 2014. In A. H. Fine (Ed.), Handbook on animal-assisted therapy: Foundations and guidelines for animal-assisted interventions ( 4th ed.) (pp. 415-418). San Diego, CA: Elsevier Academic Press. 
Lord, C., Rutter, M., \& Le Couteur, A. (1994). Autism Diagnostic Interview-Revised: A revised version of a diagnostic interview for caregivers of individuals with possible pervasive developmental disorders. Journal of Autism and Developmental Disorders, 24(5), 659685. https://doi.org/10.1007/BF02172145

Lundqvist, M., Carlsson, P., Sjödahl, R., Theodorsson, E., \& Levin, L. (2017). Patient benefit of dogassisted interventions in health care: A systematic review. BMC Complementary and Alternative Medicine, 17, 358. https://doi.org/10.1186/s12906-017-1844-7

Martin, F., \& Farnum, J. (2002). Animal-assisted therapy for children with pervasive developmental disorders. Western Journal of Nursing Research, 24(6), 657670. https://doi.org/10.1177/019394502320555403

Maujean, A., Pepping, C. A., \& Kendall, E. (2015). A systematic review of randomized controlled trials of animal-assisted therapy on psychosocial outcomes. Anthrozoos, 28(1), 2336. https://doi.org/10.2752/089279315X14129350721812

Nimer, J., \& Lundahl, B. (2007). Animal-assisted therapy: A meta-analysis. Anthrozoos, 20(3), 225238. https://doi.org/10.2752/089279307X224773

O'Haire, M. E. (2013). Animal-assisted intervention for autism spectrum disorder: A systematic literature review. Journal of Autism and Developmental Disorders, 43(7), 16061622. https://doi.org/10.1007/s10803-012-1707-5

O'Haire, M. E. (2017). Research on animal-assisted intervention and autism spectrum disorder, 2012 2015. Applied Developmental Science, 21(3), 200 216. https://doi.org/10.1080/10888691.2016.1243988

Prothmann, A., Ettrich, C., \& Prothmann, S. (2009). Preference for, and responsiveness to, people, dogs and objects in children with autism. Anthrozoös, 22(2), 161171. https://doi.org/10.2752/175303709X434185

Redefer, L. A., \& Goodman, J. F. (1989). Brief report: Pet-facilitated therapy with autistic children. Journal of Autism and Developmental Disorders, 19(3), 461467. https://doi.org/10.1007/BF02212943

Richeson, N. E., \& McCullough, W. T. (2002). An evidence-based animal-assisted therapy protocol and flow sheet for the geriatric recreation therapy practice. American Journal of Recreation Therapy, 1(1), 25-31.

Richeson, N. E., \& McCullough, W. T. (2003). A therapeutic recreation intervention using animalassisted therapy: Effects on the subjective well-being of older adults. Annual in Therapeutic Recreation, 12(1), 1-6.

Rogers, S. J., \& Vismara, L. A. (2008). Evidence-based comprehensive treatments for early autism. Journal of Clinical Child and Adolescent Psychology, 37(1), 838. https://doi.org/10.1080/15374410701817808

Sams, M. J., Fortney, E. V., \& Willenbring, S. (2006). Occupational therapy incorporating animals for children with autism: A pilot investigation. American Journal of Occupational Therapy, 60(3), 268-274. https://doi.org/10.5014/ajot.60.3.268

Sheldrick, R. C., Maye, M. P., \& Carter, A. S. (2017). Age at first identification of autism spectrum disorder: An analysis of two US surveys. Journal of the American Academy of Child and Adolescent Psychiatry, 56(4), 313- 320. https://doi.org/10.1016/j.jaac.2017.01.012

Silva, K., Correia, R., Lima, M., Magalhães, A., \& de Sousa, L. (2011). Can dogs prime autistic children for therapy? Evidence from a single case study. The Journal of Alternative and Complementary Medicine, 17(7), 655- 659. https://doi.org/10.1089/acm.2010.0436

Stevenson, K., Jarred, S., Hinchcliffe, V., \& Roberts, K. (2015). Can a dog be used as a motivator to develop social interaction and engagement with teachers for students with autism? Support for Learning, 30(4), 341-363. https://doi.org/10.1111/1467-9604.12105

Viau, R., Arsenault-Lapierre, G., Fecteau, S., Champagne, N., Walker, C. D., \& Lupien, S. (2010). Effect of service dogs on salivary cortisol secretion in autistic children. Psychoneuroendocrinology, 35(8), 11871193. https://doi.org/10.1016/j.psyneuen.2010.02.004

Virués-Ortega, J., Pastor-Barriuso, R., Castellote, J. M., Población, A., \& de Pedro-Cuesta, J. (2012). Effect of animal-assisted therapy on the psychological and functional status of elderly populations and patients with psychiatric disorders: A meta-analysis. Health Psychology Review, 6(2), 197-221. https://doi.org/10.1080/17437199.2010.534965 